THE ASTROPHYSICAL JOURNAL SUPPLEMENT SERIES, 90:869-876, 1994 February

(C) 1994. The American Astronomical Society. All rights reserved. Printed in U.S.A.

\title{
ACCELERATION PROCESSES FOR GAMMA-RAY BURSTS
}

\author{
IGOR G. MITROFANOV \\ Institute for Space Research, Moscow 117810, Russia \\ Received 1993 March 2; accepted 1993 July 21
}

\begin{abstract}
Is it shown that for those astronomical models of cosmic gamma-ray bursts (GRBs) which are associated with galactic neutron stars (NSs), the initial energy of the outburst could be converted to gamma-rays through processes of particle acceleration. The main emission mechanisms are considered for two basic alternatives, when particles are accelerated either by radiative pressure or by an electric field.
\end{abstract}

Subject headings: acceleration of particles - gamma rays: bursts - radiation mechanisms: nonthermal

\section{INTRODUCTION}

While the nature of cosmic gamma-ray bursts (GRBs) is even less clear now than it was before the launch of the Compton Gamma-Ray Observatory (e.g., see Fishman et al. 1992), more evidence has been obtained, due to the successful work of the Observatory and further studies of the data from other missions, that particle acceleration processes have to take place in the emitting sources. Indeed,

1. A lot of GRB energy spectra have power-law shapes without high-energy cutoffs up to several $\mathrm{MeV}$ or even tens of $\mathrm{MeV}$ (e.g., see Matz et al. 1985; Jourdain et al. 1992; Share et al. 1992); for some GRBs, photons with energies above $150 \mathrm{MeV}$ were detected by GRO/EGRET (Schneid et al. 1992);

2. Sub-millisecond time structure has been found for intense GRBs by GRO/BATSE (Bhat et al. 1993), both for the rise fronts and the back slopes of the time histories.

No thermal mechanism could be responsible for this emission. Relativistic beams of $e^{-}$, or possibly $e^{+}$, are the most probable emitters of gamma-rays with those properties. Therefore, some acceleration mechanism has to work in this case.

The key problem of GRBs is the astronomical origin of their sources. The isotropic distribution on the sky, but inhomogeneous distribution in space (e.g., see Meegan et al. 1992) corresponds to the following alternatives for the location of the sources:

(i) Neutron stars (NSs) from the Extended Galactic Corona with upper distances about 100-150 kpc (see Jennings 1984; Shklovskii \& Mitrofanov 1985);

(ii) NSs from the Solar Galactic Vicinity with upper distances up to $1-3 \mathrm{kpc}$ (see Mitrofanov 1993);

(iii) Some cataclysms on cosmological distances with maximal redshift $z$ about 1-2 (e.g., see Paczynski 1992).

According to a recent analysis of PHOBOS/APEX data, there is no difference in the shape of the back slopes of averaged time profiles for strong and weak events (Mitrofanov et al. 1992a, b). Therefore, the hypothesis of the cosmological origin of GRBs (iii) cannot be accepted, because of the absence of a $(1+z)$ effect in the averaged time histories.

For the other two alternatives, (i) and (ii), that associate GRBs with the Galaxy, NSs are the only population that is thought to be responsible for the GRB emission. However, the physical origin of the sporadic outbursts of NSs is the subject of numerous theoretical speculations, and all possible physical mechanisms for the emission of GRBs should be appropriately developed. In particular, the origin of GRBs could be associated with sporadic activity of dead isolated pulsars with rather strong magnetic fields (see Rudermann \& Cheng 1988; Mitrofanov 1993), that might be activated either by spontaneous glitches (e.g., see Epstein 1992), or by sporadic NS/comet encounters (e.g., see Mitrofanov \& Sagdeev 1990), or by something else. Therefore, processes of particle acceleration in gamma-ray bursts are considered below for NSs with strong magnetic fields.

\section{RADIATIVE EJECTION IN MAGNETOSPHERES OF NEUTRON STARS \\ 2.1. Radiative Force in Photosphere with Strong Magnetic Field}

The electron cyclotron resonance strongly increases the radiative force acting on a plasma in a strong magnetic field compared to the field-free case, when Thomson scattering is responsible for the radiative pressure. According to Mitrofanov \& Pavlov (1982) and Mitrofanov (1991), for a stationary electron above the photosphere, the blackbody radiative forces acting along the magnetic lines $\boldsymbol{B}$, and along the normal to the emitting surface $N$ are equal to

$$
\begin{aligned}
f_{B}=f_{\mathrm{T}} \frac{45}{24 \pi^{4} \alpha}\left(\frac{m c^{2}}{k T}\right) \frac{b^{3} \cos \theta(7-\cos \theta)}{\exp (b)-1} & \\
& \sim 800 f_{\mathrm{T}} T_{7} \frac{b^{3} \cos \theta(7-\cos \theta)}{\exp (b)-1}
\end{aligned}
$$

and

$$
f_{N}=f_{B}(\theta=0) \frac{5+\cos ^{2} \theta}{6}
$$

respectively, where

$$
f_{\mathbf{T}}=\frac{\sigma_{\mathrm{T}} \sigma_{\mathrm{SB}} T^{4}}{c}
$$


corresponds to the field-free case with Thomson cross section $\sigma_{\mathrm{T}}, T$ is the blackbody temperature, $b=\hbar \omega_{B} / k T, \theta$ is the angle between $B$ and $N, \alpha$ is the fine-structure constant, $\sigma_{\mathrm{SB}}$ is the Stephan-Boltzmann constant, and $\omega_{B}$ is the electron cyclotron frequency.

From equation (1) it is clear that the effect of "cyclotron enhancement" of radiative pressure exists, i.e., $f_{B} \gg f_{\mathrm{T}}$, provided the radiation temperature $k T$ and cyclotron energy $\hbar \omega_{B}$ are relatively close, or more precisely, provided

$$
0.1\left(\frac{k T}{m c^{2}}\right)^{1 / 2} \gg b \gg \ln \left(\frac{40 m c^{2}}{k T b^{3}}\right) .
$$

Due to the effect of "cyclotron enhancement" the critical flux of radiation $F_{\mathrm{cr}}$, for which the gravitational attraction of protons is compensated by the radiative pressure on electrons, decreases strongly in comparison with the classical Eddington value $F_{\mathrm{Ed}}$ based on the Thomson cross section $\sigma_{\mathrm{T}}$ :

$$
F_{\text {cr }} \simeq 1.3 \times 10^{-3} F_{\mathrm{Ed}} b^{-3}[\exp (b)-1] T_{7}
$$

Therefore, for the broad ranges of magnetic field strengths $10^{10}-10^{12} \mathrm{G}$ and temperatures of blackbody emission around $10^{7} \mathrm{~K}$, that are thought to be quite natural for outbursing NSs (see Fig. 1), the critical value $F_{\mathrm{cr}}$ is much smaller than $F_{\mathrm{Ed}}$. Consequently, the possibility of the radiatively driven ejection of plasma should be taken into account when modeling of GRB sources.

In the field of theoretical studies of GRBs, there are wellknown papers that have considered the up-scattering of photons by outgoing relativistic particles in the magnetosphere of a

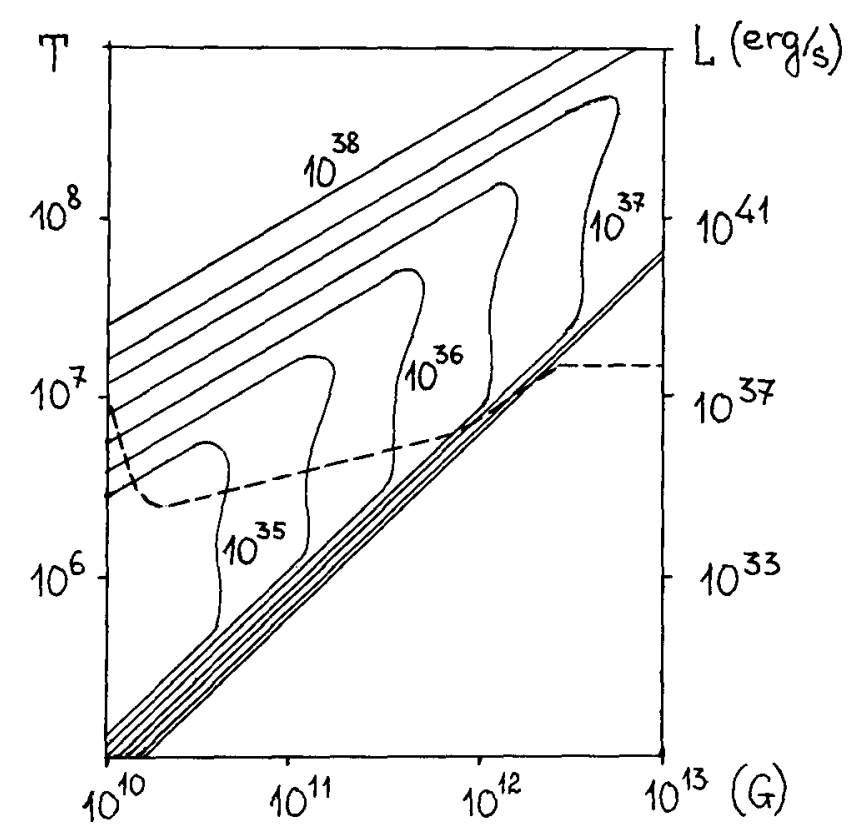

Fig. 1.-Values of temperature and magnetic field are presented (Mitrofanov 1987) for which the effect of "cyclotron enhancement" occurs (the NS luminosities corresponding to these temperatures are indicated at the right). Levels of critical luminosity are indicated by solid lines. Above the dotted line the luminosity of NS with homogeneous brightness prevails over the critical values. neutron star (e.g., see Dermer 1989, 1990; Vitello \& Dermer 1991). In these models, particles with specified power-law energy distributions were thought to be initially ejected into the magnetosphere by some independent acceleration mechanism, and their energy losses due to up-scattering of photons were taken into account. In this section the complementary physical case is considered, where outgoing particles are accelerated up to equilibrium Lorentz-factors by the pressure of the supericritical radiation flux from the heated surface of a neutron star (Mitrofanov \& Pavlov 1982; Mitrofanov \& Tsygan 1982; Mitrofanov 1984; Chernenko \& Mitrofanov 1993).

\subsection{Boundary Instability of the Magnetized Photosphere}

The radiative force (1) has been estimated above the photosphere using a smooth blackbody spectrum. However, in more realistic cases, the contribution of the cyclotron resonance in the scattering cross section to the radiative pressure could be almost totally compensated, because the thermal radiation in the magnetized photosphere should be strongly depressed by the resonant cyclotron absorption (Gnedin \& Nagel 1984). However, even in this case the effect of "cyclotron enhancement" might manifest itself, giving rise to instabilities of the boundary layer of the NS's photopshere (Mitrofanov 1987).

The first one is the "pulsation instability," which might grow from infinitely small pulsations of the boundary layer (Fig. 2). These pulsations lead to regular Doppler shifts of the cyclotron resonance for scattering in the boundary layer relative to the stable position of the cyclotron resonance for absorption. If at $\omega \sim \omega_{B}$ the photospheric continuum rises with increasing energy, the "pulsation instability" is shown to have a positive increment (Mitrofanov 1987). When the amplitude of the pulsations becomes sufficiently large, i.e., when the Doppler shift exceeds the spectral width of the resonant absorption, the average radiative force acting on the layer becomes comparable to the force (1), which was estimated earlier for a smooth blackbody spectrum.

Another one is the "expansion instability." Because the magnetic field is frozen into the plasma, any expansion/compression of the boundary layer leads to red/blueshifts of the position of the cyclotron resonance for the scattering in this layer compared to the stable position of the resonance absorption (Fig. 3). If at $\omega \sim \omega_{B}$ the photospheric continuum decreases with increasing energy, at each cycle of expansion/compression the amplitude of the oscillation rises (Mitrofanov 1987). Again, when the scattering resonance becomes shifted well outside the width of the resonance absorption, the "cyclotron enhancement" of the radiative pressure (1) has the full strength.

Therefore, if the flux of photospheric blackbody emission is depressed at the cyclotron resonance, but the corresponding continuum exceeds the critical value $F_{\text {cr }}(5)$, the radiatively driven ejection of the photospheric plasma could be initiated either by "pulsation" or by "expansion" instabilities.

\subsection{Radiatively Driven Relativistic Ejection of Photospheric Plasma}

Let us consider the radiative ejection of plasma with negligible opacity above a hot spot with supercritical blackbody emission on the magnetic pole of a NS, $R_{\mathrm{sp}} \ll R_{\mathrm{NS}}$, assuming the 

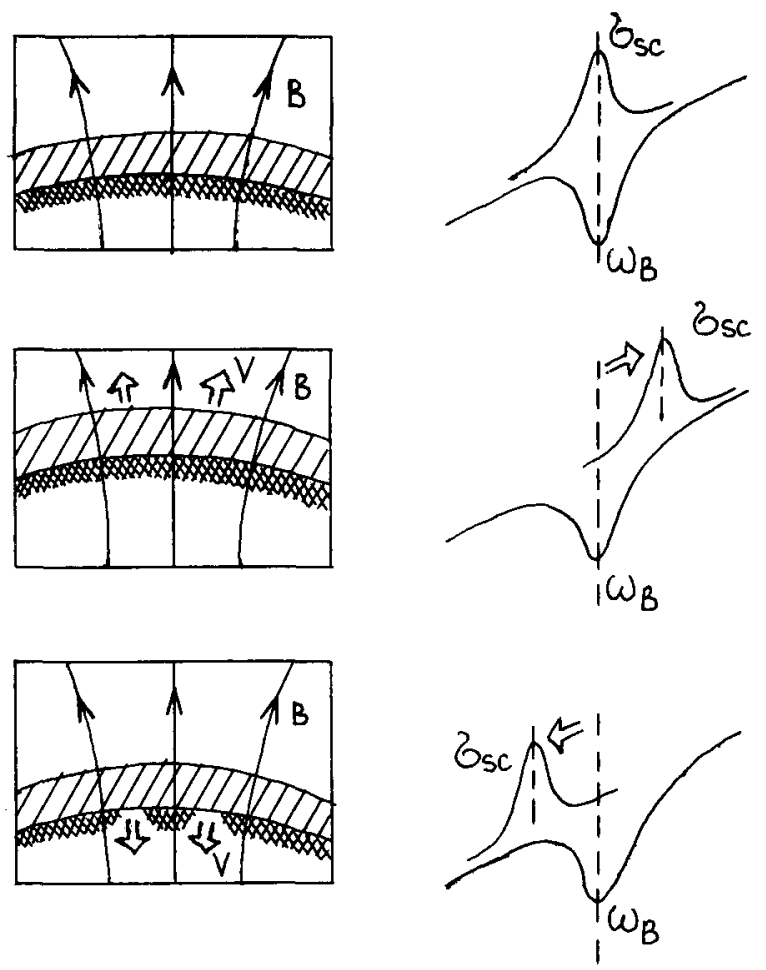

Fig. 2.-Physical reason for "pulsation instability." When layer goes up, resonance in the scattering layer shifts to the blue wing of absorption; when it goes down, resonance shifts to the red wing. For radiation with a rising spectrum, the pulsation amplitude increases for each cycle.

density of radiative energy $W_{r}$ is much smaller than the density of magnetic energy $W_{\mathrm{B}}$ (Mitrofanov \& Tsygan 1982; Chernenko \& Mitrofanov 1993). Therefore, at each point, the velocity of the ejecting plasma should be parallel to the magnetic field line. In the supercritical radiation, there is an equilibrium Lorentz-factor of the driven plasma for which the total radiative force is 0 . There are two modes of ejection, when the equilibrium Lorentz-factors correspond to different conditions.

In the first case (Fig. 4, mode $I$ ), the radiative force is produced by incoming photons with a broad set of directions, including some moving along the magnetic/velocity vector. For this mode, there will be no overall radiative force when the net momentum from the incoming accelerating photons (in the comoving frame they come from the lower hemisphere) is totally compensated by the net momentum from decelerating photons (which come from the upper hemisphere in the comoving frame). The equilibrium Lorentz-factor is $\gamma_{\mathrm{eq}}=\gamma_{\mathrm{kin}}$, where $\gamma_{\text {kin }}$ is estimated by integrating equation (1) for the radiative force over all angles and energies of the incoming photons, taking into account their polarization.

If the ejected plasma is close to the center of the radiating spot (Fig. $4 a$, mode $I$ ), one can approximately use an infinite radiating plane. Above the infinite plane with blackbody emission, the equilibrium Lorentz-factor $\gamma_{\mathrm{kin}}$ is as small as 1.4 both with and without a magnetic field. However, in the magnetic field $\gamma_{\mathrm{kin}}$ could be rather large, even as large as infinity, in two cases: either if the spectrum of radiation rises with increasing energy faster than $\propto \omega^{3}$ (Table 1), or if the radiation has strong circular polarization (Table 2). Of course, the radiation from the photosphere hardly has the spectrum with this power law: the thermal-like emission is much more probable in this case. However, in the presence of the strong magnetic field the thermal emission from the photosphere might have high linear and/or circular polarization. Therefore, very large values of the equilibrium Lorentz-factor could not be fully excluded even in the thermal radiation field.

In the second case, when the ejected plasma is far from the spot (see $I$ in Fig. $4 b$ ), the equilibrium Lorentz-factor $\gamma_{\text {kin }}$ is determined mainly by the narrow angular distribution of photons from the hot spot. Naturally, $\gamma_{\text {kin }}$ increases with increasing distance from the spot (Table 3). However, for different spectra and polarizations of the accelerating radiation the growth of $\gamma_{\text {kin }}$ with distance could be rather different.

In the second mode $I I$ there are no photons moving along the velocity of the ejected particles, i.e., along the field line (Fig. 4). In this mode the equilibrium Lorentz-factor $\gamma_{\mathrm{eq}}=$ $\gamma_{\text {geom }}$ corresponds to the purely geometrical condition that in the comoving frame the net radiative force is perpendicular to the field line. Therefore, for the distant limit (see $I I$ in Fig. $4 b$ ), the value of the Lorentz-factor does not depend either on the spectrum or on the polarization of the incident radiation and is equal to $\gamma_{\text {geom }}=\left(1-x_{0}\right)^{-1 / 2}$, where $x_{0}$ is cos of the angle between the direction of radiation from the spot and the line of the local magnetic field.

These two modes of radiative ejection, $I$ and $I I$, have been compared numerically for two sets of parameters: (1) for the case of extremely high temperature and relatively low field $T=$ $10^{9} \mathrm{~K}$ and $B=10^{11} \mathrm{G}$, and (2) for the case of rather low temper-
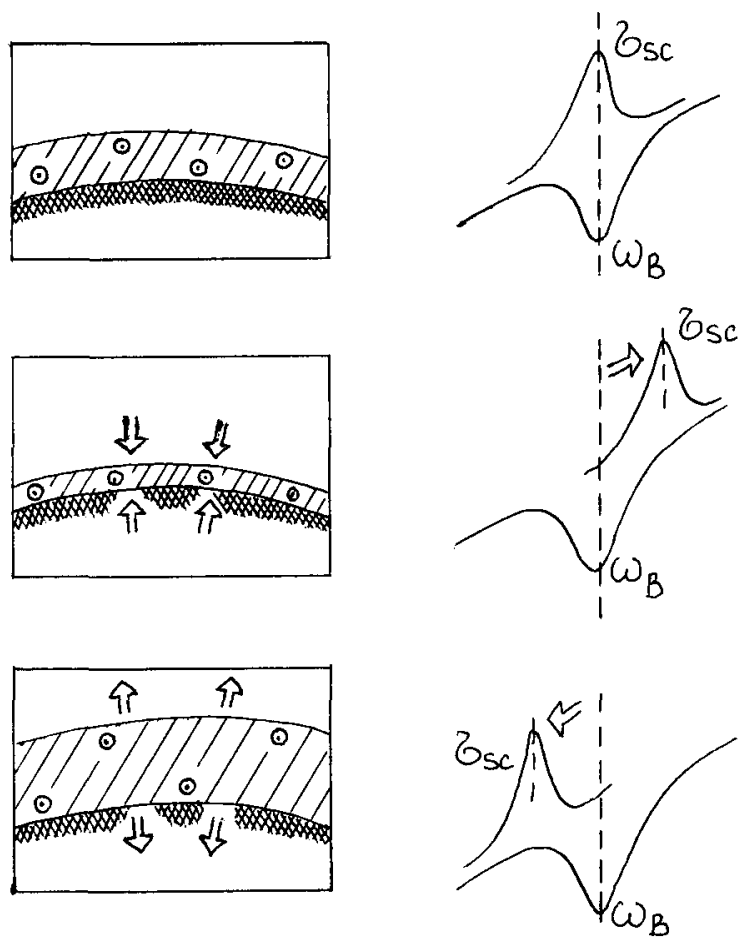

Fig. 3.-Physical reason for "expansion instability." When layer compresses, resonance in the scattering layer shifts to the blue wing of absorption; when it expands, resonance shifts to the red wing. For radiation with a decreasing spectrum the pulsation amplitude increases for each cycle. 

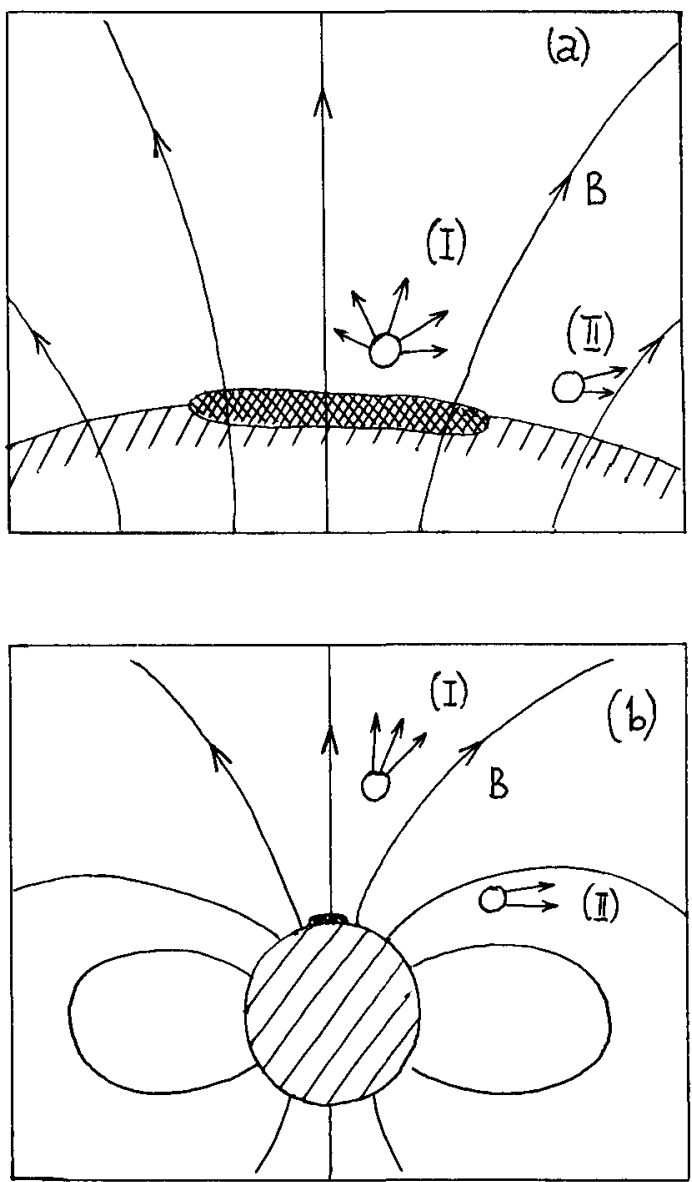

FIG. 4.-Two modes of radiative ejection in close $(a)$ and distant $(b)$ positions from radiating spot.

ature but stronger field $T=10^{7} \mathrm{~K}$ and $B=10^{12} \mathrm{G}$ (Figs. 5, 6). There is an evident difference between Lorentz-factors $\gamma_{\text {kin }}$ above the spot, but values of $\gamma_{\text {geom }}$ are quite similar well outside from it.

When considering the radiatively driven ejection of plasma, one has to take into account that heavy protons (and/or ions) are accelerated due to the electrostatic coupling with light radiatively ejected electrons. When electrons go up above the boundary of the photosphere, the negatively charged layer creates an electrostatic field. When the field of the layer be-

TABLE 1

DEPENDENCE OF $\gamma_{\text {kin }}$ ON THE SPECTRAL INDEX OF RADIATION $\propto \omega^{n}$ (INFINITE PLANE, ZERO POLARIZATION)

\begin{tabular}{lc}
\hline \hline Spectral Index $n$ & Lorentz-Factor $\gamma_{\text {kin }}$ \\
\hline $2.0 \ldots \ldots \ldots \ldots \ldots$ & 1.4 \\
$2.2 \ldots \ldots \ldots \ldots \ldots$ & 1.5 \\
$2.4 \ldots \ldots \ldots \ldots \cdots$ & 1.6 \\
$2.6 \ldots \ldots \ldots \ldots \ldots$ & 1.8 \\
$2.8 \ldots \ldots \ldots \ldots$ & 2.3 \\
$3.0 \ldots \ldots \ldots \ldots$ & $\infty$ \\
\hline
\end{tabular}

TABLE 2

DEPENDENCE OF $\gamma_{\text {kin }}$ ON LiNEAR $P_{l}$ AND CiRCULAR $P_{q}$ Polarization (INFINITE PLANE, RAYLEIGH-JEANS SPECTRUM)

\begin{tabular}{cccc}
\hline \hline$P_{l}$ & $P_{q}=+\left(1-P_{l}^{2}\right)^{1 / 2}$ & $P_{q}=0$ & $P_{q}=-\left(1-P_{l}^{2}\right)^{1 / 2}$ \\
\hline$-1.0 \ldots \ldots \ldots$ & 1.53 & 1.53 & 1.53 \\
$-0.6 \ldots \ldots \cdots$ & 6.50 & 1.50 & 1.06 \\
$-0.3 \ldots \ldots \ldots$ & 212.05 & 1.45 & 1.03 \\
$0 \ldots \ldots \ldots \cdots$ & $\infty$ & 1.42 & 1.05 \\
$0.3 \ldots \ldots \ldots$ & 24.46 & 1.40 & 1.09 \\
$0.6 \ldots \ldots \cdots$ & 2.73 & 1.39 & 1.14 \\
$1.0 \ldots \ldots \cdots$ & 1.37 & 1.37 & 1.37 \\
\hline
\end{tabular}

comes equal to $f_{B} / e$, new electrons do not escape from the photosphere because the radiative pressure is compensated by the electrostatic repulsion. However, the electric field of the escaping electrons pulls out heavy particles, and the positively charged layer follows the layer of electrons. The net electric field becomes zero on the surface, and the next layer of electrons is radiatively ejected from the surface.

Thus, the radiatively driven ejection of plasma has to be accompanied by Langmuire-like pulsations associated with the electric field:

$$
E \sim f_{B} / e=3 \times 10^{3} T_{7} b^{3}[\exp (b)-1]^{-1} \mathrm{~V} \mathrm{~cm}^{-1},
$$

which is sufficient to accelerate heavy particles up to energies about $\gamma_{\text {kin }} m_{p} c^{2} \sim 10^{3} \mathrm{MeV}$ along a path about $3 \times 10^{5} \mathrm{~cm}$.

Along this path from the surface of the NS, which is less than $R_{\mathrm{NS}}$, each pair of negative and positive layers has to join into one neutral plasma layer ejected with the Lorentz-factor $\gamma_{\mathrm{eq}}$ along the field lines. Further radiative acceleration of the ejected plasma with increasing distance from the surface could be associated with a small separation of the electrons and ions along the magnetic field lines. The corresponding electric field is much smaller than the one estimated above in equation (6), but it acts along a much longer path $\left(\gg R_{\mathrm{NS}}\right)$.

Finally, the radiatively accelerated ejection with $\gamma=\gamma_{\text {eq }}$ has to finish at some distance from the photosphere, when the radiative force becomes so small that its work along the path does not provide any growth in the particle's energy. After that, free ejection of the plasma is thought to occur without any further acceleration.

Of course, the regime of radiative ejection could be rather different if the plasma has a noticeable cyclotron opacity. In the case $I$ (see above) ejected plasma would attain higher Lo-

TABLE 3

DEPENDENCE OF $\gamma_{\text {kin }}$ AND $\Gamma_{\text {kin }}$ ON $\zeta$ ABOVE THE CENTER OF THE Hot SPOT (BlaCkBody SPECTRUM, Zero Polarization)

\begin{tabular}{|c|c|c|c|c|c|c|}
\hline \multirow[b]{2}{*}{5} & \multicolumn{3}{|c|}{$\gamma_{\text {kin }}$} & \multicolumn{3}{|c|}{$\Gamma_{\text {kin }}$} \\
\hline & $b=0.01$ & $b=0.1$ & $b=1.0$ & $b=0.01$ & $b=0.1$ & $b=1.0$ \\
\hline 0.0 & 1.7 & 1.6 & 1.4 & 5.6 & 5.3 & 3.9 \\
\hline 1.0 & 2.8 & 2.7 & 2.1 & 5.3 & 5.0 & 3.4 \\
\hline 10.0 & 20.4 & 16.2 & 10.1 & 5.1 & 3.6 & 2.0 \\
\hline
\end{tabular}




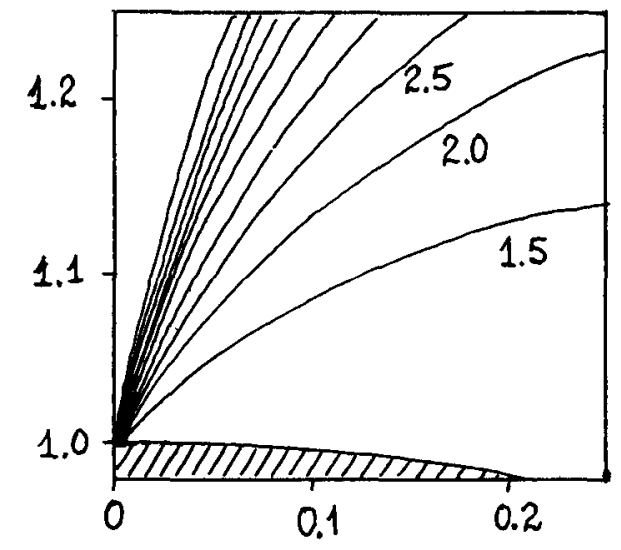

FIG. 5.-Sections of axially symmetric surfaces of equal $\gamma_{\text {kin }}$ in one quadrant, when a point source (radiating spot) is located on the magnetic pole (Chernenko \& Mitrafanov 1993). Dimensions are in units $R_{\mathrm{NS}}$

rentz-factors $\gamma_{\text {kin }}$, because in this case the lower layer does screen some part of the decelerating photons, while it does not interact at all with the accelerating ones (Mitrofanov \& Tsygan 1982). For plasma with a noticeable cyclotron opacity, values of $\gamma_{\text {kin }}$ could be several times higher.

\subsection{Drift of Radiatively Ejected Plasma Across the Field Lines}

According to $\S 2.1$, in the magnetic field the mean radiative force $f$ has a component $f_{\perp}$ perpendicular to the magnetic field $B$. While the plasma is ejected by a radiative force $f_{\|,}$, the drift of electrons has to occur across $N$ and $B$ with the drift velocity

$$
V_{\mathrm{dr}}=\frac{f_{\perp} c^{2}}{e B} .
$$

The velocity $V_{\mathrm{dr}}$ depends on the ratio between the densities of radiative and magnetic energy, $W_{r}$ and $W_{B}$, respectively:

$$
V_{\mathrm{dr}}=c \frac{\alpha}{3} \frac{f_{\perp}}{f_{\mathrm{T}}} \frac{W_{r}}{W_{B}} \frac{B}{B_{c}},
$$

where $B_{c}$ is the critical magnetic field $B_{c}=m^{2} c^{3} / e \hbar=4.4 \times$ $10^{13} \mathrm{G}$. On the contrary to the previous $\S 2.3$, where $W_{r} \ll W_{B}$ has been assumed, a more general case $W_{r} \leq W_{B}$ is considered below.

In the unstable boundary layer of the magnetized photosphere (see $\S 2.2$ ) the drift of ejected plasma generates a current, which induces a perturbation of the local magnetic field:

$$
\Delta B \sim B \frac{W_{r}}{W_{B}} .
$$

If the ejection occurs from the whole surface of the NS, electrons in the outflowing plasma undergo an azimuthal drift, which generates a global current and perturbs the dipole magnetic field. If the magnetosphere is full of ejected plasma with density $\rho_{\mathrm{ej}}$, the induced magnetic moment could be estimated by

$$
\Delta \mu \sim \frac{e}{2 m_{p} c} \rho_{\mathrm{ej}} V_{\mathrm{dr}} R_{\mathrm{NS}}^{4} \sim \mu_{\mathrm{NS}} \tau_{\mathrm{T}} \frac{f_{\perp} W_{r}}{f_{\mathrm{T}} W_{B}}
$$

where $\tau_{\mathrm{T}}=\left(\rho_{\mathrm{ej}} / m_{p}\right) \sigma_{\mathrm{T}} R_{\mathrm{NS}}$ is the optical thickness of the ejected plasma and $\mu_{\mathrm{NS}}$ is magnetic dipole moment of the NS.

Pulsations of the electrostatic field on the surface of the NS (see $\S 2.3$ ) should lead to pulsations of the radiatively driven drift along the surface layer. The same pulsation should be associated with the induced current and magnetic field.

Therefore, the radiative drift of electrons in the radiatively ejected plasma provides the physical connection between the outgoing radiation, accelerated particles, and magnetic field.

\subsection{Outbursts of Neutron Stars: Coupling between Radiative and Magnetic Fields}

One might consider the outburst of a NS, when the energy is supplied either to the local heated spot or to the total photosphere. There are two possible mechanisms for such an energization, i.e., accretion onto the surface and thermonuclear burning inside it (e.g., see Woosley 1984; Lasota et al. 1992). On the other hand, mechanisms for the direct energization of the magnetosphere of the NS could also lead to the local heating on the surface due to the action of relativistic particles and hard photons (Mitrofanov 1984).

Therefore, taking into account the strong gravitational field of the NS, the radiation field with extremely high energy density $W_{r}$ and finally the strong magnetic field with energy density $W_{B}$, one might conclude that the physics of GRBs could be determined by two physical conditions:

The first condition represents the ratio between the gravitational attraction on heavy particles and the radiative pressure on electrons (see $\S 2.1$ ). Applying the estimate of the radiative force equation (1) to the data on the real GRBs, the critical distance to the source $d_{0}$, which corresponds to $f_{\mathrm{gr}}=f_{\text {rad }}$, could be estimated

$$
d_{0} \simeq 3\left(\frac{10^{-3} \mathrm{ergs} \mathrm{cm}^{-2} \mathrm{~s}^{-1}}{F_{\mathrm{GRB}}}\right)^{1 / 2}\left(\frac{R_{\mathrm{sp}}}{10^{5} \mathrm{~cm}}\right) \mathrm{pc}
$$

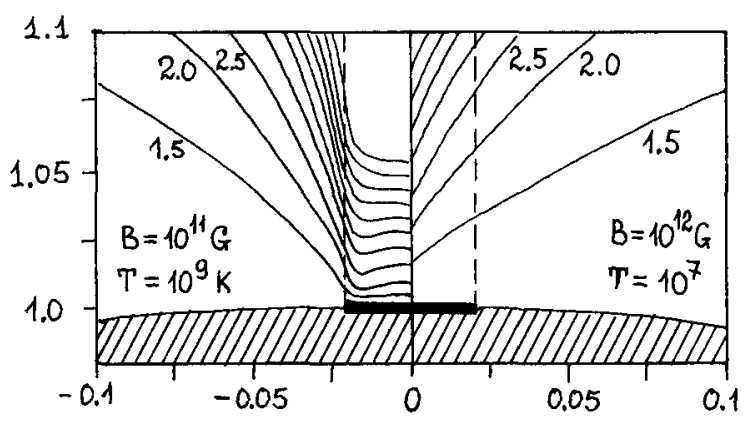

FIG. 6.-Zoom-in of Fig. 5: distribution of $\gamma_{\text {kin }}$ near the hot spot. Regions inside and outside dashed lines corresponds to $\gamma=\gamma_{\mathrm{kin}}$ and $\gamma=\gamma_{\mathrm{geom}}$, respectively (Chernenko \& Mitrofanov 1993). 
where $F_{\mathrm{GRB}}$ is the energy flux of strong events. The similar estimations have been obtained for the specific case of strong event GB 880205 (Lamb et al. 1990; Mitrofanov 1991) with double-component depression in the energy spectrum, which has been identified with the first and the second harmonics of the electron cyclotron resonance.

For $d>d_{0}$ one obtains $f_{\mathrm{gr}}<f_{\mathrm{rad}}$, and radiation dominates over the gravitational force. The boundary of the photosphere becomes unstable (see $\S 2.2$ ), and radiatively driven ejection has to occur from the surface of the magnetized NS (see $\S 2.3$ ). The main physical effects of the radiative ejection have to take place in this case, such as Compton up-scattering, Langmuirlike pulsations, drift of ejecting plasma, and radiative/magnetic coupling.

For any models that associate GRBs with NSs, the distances to the sources of strong events are thought to be $\gg d_{0}$ (Mitrofanov 1993). Therefore, one could conclude that the radiatively driven ejection is a necessary process, which should be taken into account. A theoretical model might combine the complexity of different physical processes, but the radiative ejection should be the principal one among them.

The second condition is determined by the ratio between the densities of radiative and magnetic energies, namely by $W_{r} /$ $W_{B}$, which also depends on the distance to the NS. It is about 1 provided $d=d_{1}$, where

$$
d_{1} \simeq 30\left(\frac{10^{-3} \mathrm{ergs} \mathrm{cm}^{-2} \mathrm{~s}^{-1}}{F_{\mathrm{GRB}}}\right)^{1 / 2}\left(\frac{R_{\mathrm{sp}}}{10^{5} \mathrm{~cm}}\right) B_{12} \mathrm{kpc} .
$$

For $d_{0}<d<d_{1}$, the radiatively driven ejection occurs along dipole field lines with an equilibrium Lorentz-factor corresponding either to $\gamma_{\mathrm{kin}}$ or to $\gamma_{\mathrm{geom}}$. Compton up-scattering of the photospheric photons might be responsible for the generation of gamma-rays in this case. The parameter $\Gamma=2 \gamma^{2}(1-$ $\beta x_{0}$ ) describes the maximal increase in energy of an up-scattered photon above a hot spot, where $x_{0}$ is cos of the maximal angle between the direction of the incoming radiation and the field line. Above the center of the spot $x_{0}(\zeta)=\zeta\left(1+\zeta^{2}\right)^{-1 / 2}$, where $\zeta=z / R_{\mathrm{NS}}$ is the dimensionless height above the surface of the spot. Values of $\gamma_{\text {kin }}$ and $\Gamma_{\text {kin }}$ are presented in Table 3 for this case.

On the other hand, far away from the spot, where the equilibrium Lorentz-factor corresponds to the geometrical condition $\gamma_{\text {geom }}($ see $\S 2.3)$, the value of $\Gamma_{\text {geom }}=2$.

For $d>d_{1}$ the magnetic field is dominated by the radiative flux. In this case, the radiatively ejected plasma moves radially from the source along straightened magnetic field lines. There are no conditions for geometrical equilibrium of the Lorentzfactor in this case, and the upper limit to energies of the upscattered photons could be associated with $\Gamma_{\text {kin }}$ only.

To calculate accurately the spectrum of up-scattered radiation, one needs to solve a self-consistent problem of radiatively driven ejection, where the energy/angle distributions of the scattered outgoing photons are calculated consistently with the energy distribution of the ejected electrons. However, for qualitative estimates of the energies of up-scattered photons, the thin plasma limit might be used, as shown above, where any influence of the scattering is ignored on the energies and angles of accelerating radiation. Inside the optically thin plasma, en- ergies of up-scattered photons could be increased 2-5 times for the "kinetic" mode and 2 times for the "geometric" mode of ejection. Therefore, up-scattering on the radiatively driven, optically thin ejecting plasma cannot explain the spectra of GRBs at high energies. Further studies of the self-consistent, optically thick case will be presented elsewhere.

\section{ELECTRIC ACCELERATION AND RADIATIVE BRAKING IN MAGNETOSPHERE OF NEUTRON STARS}

\subsection{Radiative Braking in Strong Magnetic Field}

Let us assume that the outburst of the NS happens due to the input of energy directly into the magnetosphere. In this case, gamma-rays could be generated by some electromagnetic process in the excited magnetosphere, when an electric field is generated with $(\boldsymbol{E} \cdot \boldsymbol{B}) \neq 0$. The excitation could take place either due to a spontaneous stellar quake (e.g., see Epstein 1992), or due to a NS/comet encounter (e.g., see Mitrofanov \& Sagdeev 1990).

The magnitude of the electric field in the excited magnetosphere of a rotating/vibrating NS is about

$$
\Delta E \approx 3 \times 10^{14} \xi B_{12} \mathrm{~V} \mathrm{~cm}^{-1}
$$

where $\xi$ depends on the specific mechanism of excitation. In the case of rotation, the coefficient $\xi$ equals $\left(2 R_{\mathrm{Ns}} / P c\right)^{q}$, where $P$ is the period of rotation, and $q$ is different for different models of the magnetosphere. For the vacuum case, $q=1$ and $\xi=$ $10^{-3}(0.2 s / P)$. On the other hand, for the case of vibrations the coefficient $\xi$ is estimated to be $10^{-4}$ (Smith \& Epstein 1992).

The electric field accelerates charged particles along magnetic field lines. A NS with an excited magnetosphere might have either a heated surface or heated spots on the surface due to a flux of particles and photons going down from the magnetosphere. The temperature $T$ of blackbody emission from the surface could be rather small, $k T \ll \hbar \omega_{B}$. The radiative action on the electrons could therefore be neglected, and the electrostatic acceleration should be dominant in this case, because the radiative flux would be relatively small and the effect of "cyclotron enhancement" could be neglected.

However, with increasing Lorentz-factor $\gamma$, the radiative braking increases. For the usual Thomson scattering and

$$
\gamma \ll \gamma_{\mathrm{K}-\mathrm{N}}=m c^{2} / k T=6 \times 10^{3} T_{6}^{-1}
$$

this braking corresponds to

$$
f_{\mathrm{br}}^{\mathrm{T}} \sim \frac{1}{3} f_{\mathrm{T}} \gamma^{2}
$$

and for Klein-Nishina scattering, when $\gamma>\gamma_{\mathbf{K}-\mathrm{N}}$,

$$
f_{\mathrm{br}}^{\mathrm{K}-\mathrm{N}} \sim f_{\mathrm{T}} \gamma_{\mathrm{K}-\mathrm{N}}^{2} \ln \left(\frac{2 \gamma}{\gamma_{\mathrm{K}-\mathrm{N}}}\right)
$$

Moreover, at a Lorentz-factor $\gamma_{b}=\hbar \omega_{B} / k T=100 B_{12} T_{6}^{-1}$ in the comoving frame of the electron, the energy of the scattered photons becomes equal to the electron cyclotron resonance. At this Lorentz-factor, the radiative force strongly increases due 
to the "cyclotron enhancement" (Kardashev, Mitrofanov, \& Novikov 1984), and the maximum radiative braking is

$$
f_{\mathrm{br}}^{B} \sim 2 \times 10^{7} f_{\mathrm{T}} B_{12} T_{6}^{-2}
$$

The cyclotron resonance dominates in the radiative braking provided $f_{\mathrm{br}}^{B}>f_{\mathrm{br}}^{\mathrm{K}-\mathrm{N}}$ (Fig. 7). It corresponds to the condition $B>$ $B_{*} \sim 10^{12} \mathrm{G}$.

The radiative braking might totally compensate the electrostatic acceleration if $e E<f_{\mathrm{br}}^{(\max )}$. According to Mitrofanov (1984), this condition corresponds to

$$
\Delta E \leq 10^{9} B_{12} T_{8}^{2} \mathrm{~V} \mathrm{~cm}^{-1} \text {. }
$$

Therefore, in the excited magnetosphere of the NS, electrostatic power could be directly transformed to gamma-rays by photon up-scattering at the cyclotron resonance, provided

$$
\xi<3 \times 10^{-8} T_{7}^{2}
$$

for the induced electron field (see also Smith \& Epstein 1993).

In the opposite case, $e E>f_{\mathrm{br}}^{(\max )}$, the radiative force does not compensate the electric one, and charged particles should be accelerated over the level of $\gamma_{b}$ either until they escape from the region of acceleration, or until the acceleration is compensated by the recoil from the curvature radiation or $e^{+/-}$pair creation.

\subsection{Gamma-Rays from Electrically Driven Ejection}

If the radiative braking at the electron cyclotron resonance occurs in the soft blackbody radiation field, one might expect the creation of up-scattered gamma-rays with typical energies

$$
E \sim k T \gamma_{b}^{2} \sim \frac{\left(\hbar \omega_{B}\right)^{2}}{k T} \sim 1 B_{12}^{2} T_{6}^{-1} \mathrm{MeV}
$$

A stream of relativistic particles moving along magnetic field lines could form bunches due to pulsations of the electrostatic acceleration. For example, for a vibrating NS, the electrical force is modulated by the frequency of the fundamental

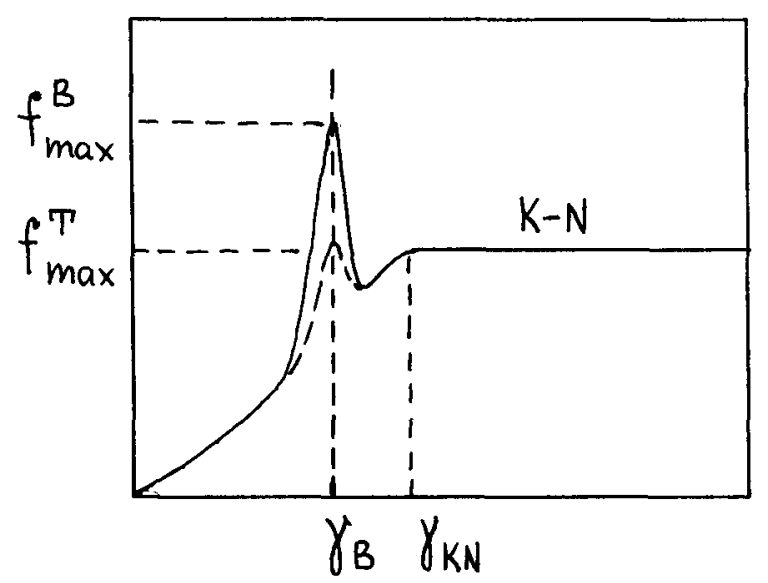

FIG. 7.-Dependence of the radiative force on Lorentz-factor of electron. Solid line corresponds to $B>B_{*}$, dashed line corresponds to $B<B_{*}$. The plateau marked by "K-N" corresponds to Klein-Nishina slope in scattering cross section. mode $\sim 10^{4} \mathrm{~Hz}$. On the other hand, MHD-waves propagating along field lines give modulations to the ejected stream. The bunches are known to exist in active magnetospheres of NSs, and they are visible as microsecond spikes in the radio data of active pulsars (Hankins 1972).

The dipole magnetic field lines of NSs are curved with radius $R_{B}>10^{6} \mathrm{~cm}$. The heated spot could be associated with a local magnetic inhomogeneity on the surface of the NS with smaller scales $10^{4}-10^{5} \mathrm{~cm}$. A relativistic bunch moving along the field lines could be observed along a tangent line of sight like a flare, with a short duration of about (Mitrofanov 1989; Chernenko \& Mitrofanov 1992)

$$
\delta t_{\text {bunch }} \sim\left(\frac{30}{\gamma}\right) \frac{10^{6} \mathrm{~cm}}{R_{B}} \mu \mathrm{S}
$$

Inside the blackbody radiation with photon number density $n_{\mathrm{ph}} \sim\left(\sigma_{S B} T^{3} / c k\right)$, a bunch of charged particles with dimension $l \sim R_{B}$ might emit photons with the maximal rate

$$
\dot{N} \sim l^{2} n_{\mathrm{ph}} c \sim 10^{42} l_{6}^{2} T_{6}^{3} \mathrm{~s}^{-1}
$$

inside the solid angle $\Omega \sim 2 \pi / \gamma^{2}$.

If it is detected by an instrument with sensitive area $S_{\text {det }}$ at a distance $d$ from the source, the microsecond bunch could be observed as a superposition of $K_{\mathrm{ph}}$ photons within several microseconds, where

$$
\begin{aligned}
K_{\mathrm{ph}} & \sim \frac{S_{\mathrm{det}} \dot{N} \delta t_{\mathrm{bunch}}}{\Omega d^{2}} \\
& \sim 5 \gamma T_{6}^{3}\left(\frac{10 \mathrm{pc}}{d}\right)^{2}\left(\frac{S_{\mathrm{det}}}{10^{3} \mathrm{~cm}^{2}}\right)\left(\frac{l}{10^{6} \mathrm{~cm}}\right) .
\end{aligned}
$$

If for some GRB $K_{\mathrm{ph}}>1$, the bunches could be observed as multiphoton counts with the mean released energy $\sim K_{\mathrm{ph}} E$ (Mitrofanov 1989). In the opposite case, no correlation would be found between counts within several microseconds in the data stream of several detectors.

\section{ASTROPHYSICAL CONCLUSIONS}

The discussed processes of particle acceleration in the magnetospheres of NSs lead to the following main conclusions:

1. The fact that the NS has a strong magnetic field leads to a considerable decrease in the value of the critical flux of radiation from the surface that is needed to compensate the gravitational attraction of the electron/proton plasma. If NSs are the sources of GRBs, the flux of emitted radiation is subcritical only for very small source distances, several parsecs. For larger distances, the sources will emit a supercritical flux of photons and, therefore, will undergo a radiatively driven ejection.

2. The relativistic Lorentz-factor of the radiatively ejected plasma is determined either by kinematic or by geometric conditions. In the case of an optically thin plasma, up-scattering of photons increases their energies by only a factor of $2-5$. Therefore the radiative ejection could not be responsible for the generation of the high-energy component of GRBs, but it might explain the production of soft gamma-rays below $\mathrm{MeV}$. 
3. If the emission in GRB is accompanied by excitations of the magnetosphere, the electric acceleration of electrons along magnetic field lines might be compensated by their braking in the soft blackbody emission above the surface. The equilibrium Lorentz-factor of the electrically driven electrons is $\sim \hbar \omega_{B} / k T$. In a magnetic field with strength greater than $10^{13}$
$\mathrm{G}$ and radiation field with temperature $0.1 \mathrm{keV}$, up-scattered photons could be generated in the broad range of energies from $1 \mathrm{MeV}$ up to $100 \mathrm{MeV}$ or even more.

The author thanks the Colloquium organizers for their financial support and kind hospitality.

\section{REFERENCES}

Bhat, P. N., et al. 1993, Nature, in press

Chernenko, A. M., \& Mitrofanov, I. G. 1992, in Proc. Los Alamos Workshop on Gamma-Ray Bursts, ed. C. Ho, R. I. Epstein, \& E. E. Fenimore (Cambridge: Cambridge Univ. Press), 55

- 1993, in Proc. Los Alamos Workshop on Isolated Pulsars, ed. K. A. van Riper, R. Epstein, \& C. Ho (Cambridge: Cambridge Univ. Press), 215

Dermer, C. D. 1989, ApJ, 347, L13

1990, ApJ, 360, 197

Epstein, R. 1992, in Proc. Los Alamos Workshop on Gamma-Ray Bursts, ed. C. Ho, R. I. Epstein, \& E. E. Fenimore (Cambridge: Cambridge Univ. Press), 1

Fishman, G. J., et al. 1992, in Proc. Huntsville Workshop on Gamma-Ray Bursts, ed. W. S. Paciesas \& G. J. Fishman (New York: AIP), 13

Gnedin, Yu. N., \& Nagel, W. 1984, A\&A, 138, 356

Hankins, T. H. 1972, ApJ, 177, L11

Jennings, M. C. 1984, in Proc. High-Energy Transients in Astrophysics, ed. S. Woosley (New York: AIP), 412

Jourdain, E., et al. 1992, in Proc. Los Alamos Workshop on Gamma-Ray Bursts ed. C. Ho, R. I. Epstein, \& E. E. Fenimore (Cambridge: Cambridge Univ. Press), 55

Kardashev, N. S., Mitrofanov, I. G., \& Novikov, I. D. 1984, Rus. Astron. J., 61,1113

Lamb, D. Q., Wang, J. C. L., \& Wasserman, I. M. 1990, ApJ, 363, 670

Lasota, J. P., et al. 1992, in Proc. Huntsville Workshop on Gamma-Ray Bursts, ed. W. S. Paciesas \& G. J. Fishman (New York: AIP), 126

Matz, S. M., et al. 1985, ApJ, 288, L37

Meegan, C. A., et al. 1992, Nature, 355, 143

Mitrofanov, I. G. 1984, Ap\&SS, 105, 245
Mitrofanov, I. G. 1987, Ap\&SS, 132, 155

1989, Ap\&SS, 155,141

1991, Ap\&SS, 176, 309

1993, in Proc. Los Alamos Workshop on Isolated Pulsars, ed. K.

A. van Riper, R. Epstein, \& C. Ho (Cambridge: Cambridge Univ. Press), 424

Mitrofanov, 1. G., et al. 1992a, in Proc. Huntsville Workshop on Gamma-

Ray Bursts, ed. W. S. Paciesas \& G. J. Fishman (New York: AIP), 163

Mitrofanov, I. G., et al. 1992b, Rus. Astron. J., 69, 1052

Mitrofanov, I. G., \& Pavlov, G. G. 1982, MNRAS, 212, 545

Mitrofanov, I. G., \& Sagdeev, R. Z. 1990, Nature, 344, 313

Mitrofanov, I. G., \& Tsygan, A. I. 1982, Ap\&SS, 84, 35

Paczynski, B. 1992, in Proc. Huntsville Workshop on Gamma-Ray Bursts, ed. W. S. Paciesas \& G. J. Fishman (New York: AIP), 144

Rudermann, M., \& Cheng, A. 1988, ApJ, 335, 306

Schaefer, B. E., et al. 1993, ApJ, 404, 673

Schneid, E. J., et al. 1992, in Proc. Huntsville Workshop on Gamma-Ray Bursts, ed. W. S. Paciesas \& G. J. Fishman (New York: AIP), 38

Share, G. H., et al. 1992, in Proc. Huntsville Workshop on Gamma-Ray Bursts, ed. W. S. Paciesas \& G. J. Fishman (New York: AIP), 32

Shklovskii, I. S., \& Mitrofanov, I. G. 1985, MNRAS, 212, 545

Smith, I. A., \& Epstein, R. I. 1992, in Proc. Los Alamos Workshop on Gamma-Ray Bursts, ed. C. Ho, R. I. Epstein, \& E. E. Fenimore (Cambridge: Cambridge Univ. Press), 29 . 1993, ApJ, 410, 315

Vitello, P., \& Dermer, C. D. 1991, ApJ, 374, 668

Woosley, S. E. 1984, in Proc. High-Energy Transients in Astrophysics, ed. S. Woosley (New York: AIP), 485 